\title{
Staged repair of truncus arteriosus with double aortic arch, tracheoesophageal fistula, and choanal atresia via a multi- disciplinary approach: a case report
}

\author{
Aditya Sengupta $^{1}$, Raghav A. Murthy ${ }^{2}$, Peter Pastuszko ${ }^{2}$ \\ ${ }^{1}$ Department of Cardiovascular Surgery, The Mount Sinai Hospital, New York, NY, USA; ${ }^{2}$ Division of Pediatric Cardiac Surgery, Department of \\ Cardiovascular Surgery, The Mount Sinai Hospital, New York, NY, USA \\ Correspondence to: Aditya Sengupta, MD. Department of Cardiovascular Surgery, The Mount Sinai Hospital, 1190 Fifth Avenue, Box 1028, New York, \\ NY 10029, USA. Email: Aditya.Sengupta.ISMMS@gmail.com.
}

\begin{abstract}
The previously unreported case of a child with an exceedingly rare amalgamation of complex defects, including truncus arteriosus (TA), double aortic arch (DAA), tracheoesophageal fistula, and choanal atresia is presented. First, on day-of-life (DOL) 2, with a joint effort involving Pediatric Cardiac Surgery, General Surgery, and Otolaryngology, division of tracheoesophageal fistula and repair of esophageal atresia, along with choanal atresia repair, was carried out. Via a right thoracotomy, the tracheoesophageal fistula, located medial to the azygous vein, was skeletonized and ligated. The proximal esophagus was then mobilized up to the thoracic inlet as it coursed through the vascular ring. This enabled esophageal anastomosis with preservation of both aortas. Next, on DOL 11, the child underwent TA repair. Following a standard midline sternotomy and cooling to moderate hypothermia, the left aortic arch was divided and oversewn. The aorta was then transected anteriorly, and the main pulmonary artery (MPA) exiting the posterior aorta was harvested as a single button. The aortic defect from the pulmonary artery button was repaired with autologous pericardium. Next, through a right ventriculotomy, the previously seen conoventricular septal defect was identified and closed. Finally, a $10-\mathrm{mm}$ pulmonary homograft was anastomosed to the pulmonary artery bifurcation to complete the repair. The patient was discharged on DOL 78 and was noted to be doing well at 1 -year follow-up. This case validates the feasibility of fistula repair complicated by DAA through a right thoracotomy, the durability of staged, complete repair of TA and DAA, and the advantages of a holistic, team-based approach that optimizes timing of all repairs based upon a careful consideration of the exponential, rather than additive, effects of multi-organ disease on post-cardiac surgery outcomes in neonates.
\end{abstract}

Keywords: Truncus arteriosus (TA); double aortic arch (DAA); tracheoesophageal fistula; thoracotomy; case report

Submitted Jul 03, 2020. Accepted for publication Oct 04, 2020.

doi: $10.21037 /$ tp-20-174

View this article at: http://dx.doi.org/10.21037/tp-20-174

\section{Introduction}

Truncus arteriosus (TA) is occasionally associated with right aortic arch (RAA) or aortic arch (AA) interruption, but very uncommonly with double aortic arch (DAA) $(1,2)$. A previously unreported case of a child with an exceedingly rare amalgamation of complex defects, including TA, DAA, tracheoesophageal fistula (TEF), and choanal atresia (CA), managed with a staged, multi-disciplinary approach, is presented in accordance with the CARE reporting checklist (available at http://dx. doi. org/10. 21037/tp-20-174).

\section{Case presentation}

A $2.96-\mathrm{kg}$ female with severe immunodeficiency, Dandy- 

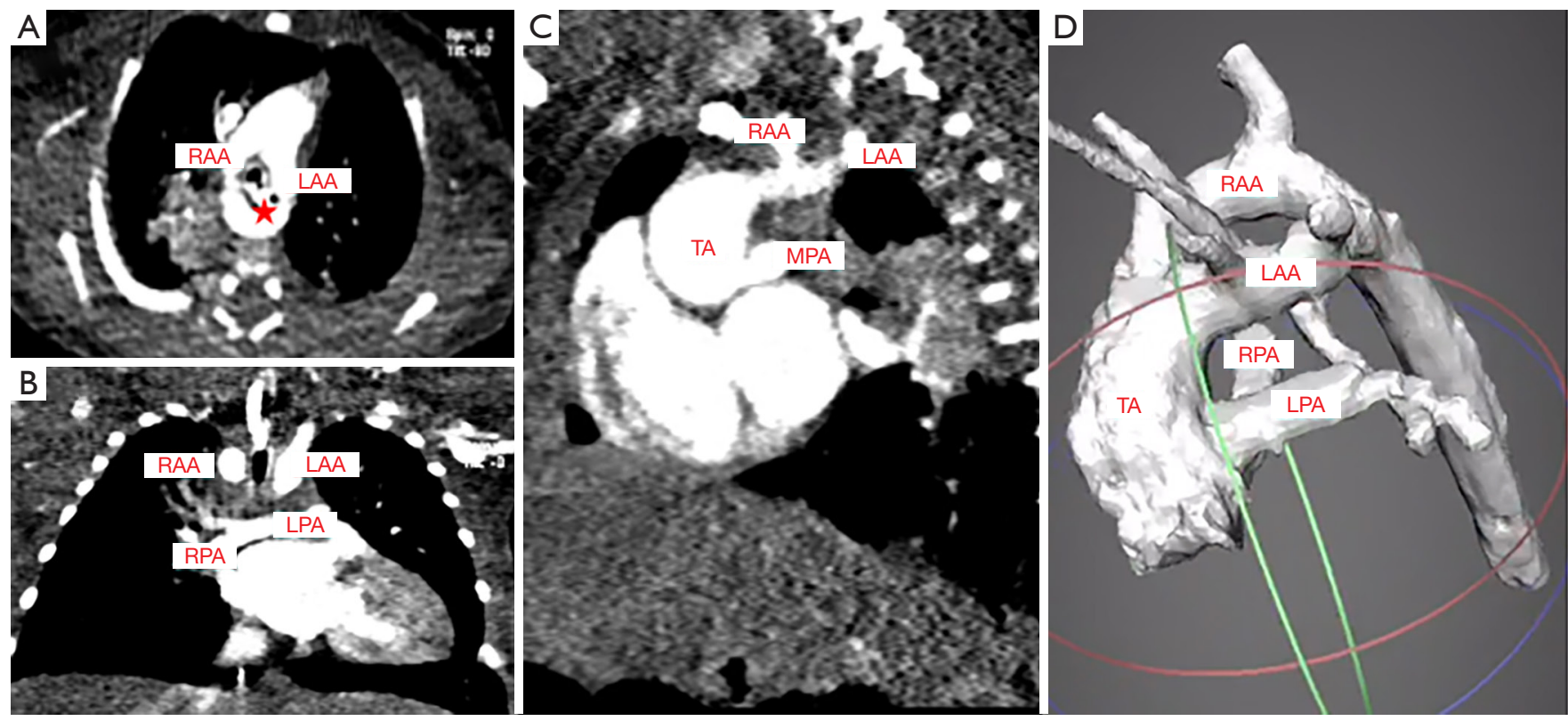

Figure 1 Preoperative computed tomography angiography. Preoperative CTA in the (A) transverse, (B) coronal, (C) sagittal, and (D) 3D reconstructed views shows truncus arteriosus with a sub-truncal VSD with a single outflow, a very short MPA arising from the truncal root posteriorly and leftward with quick bifurcation into the branch PAs, a double aortic arch encircling the trachea and esophagus, and the and the proximal esophageal pouch (asterisk) coursing to the left of the subclavian vein and through the vascular ring. CTA, computed tomography angiography; LAA, left aortic arch; LPA, left pulmonary artery; MPA, main pulmonary artery; RAA, right aortic arch; RPA, right pulmonary artery; VSD, ventricular septal defect.

Walker malformation, and prenatally-diagnosed type 1 TA and DAA (RAA >left aortic arch, or LAA), was found to have CA and TEF with proximal esophageal atresia (Figure 1). On day-of-life (DOL) 2, with a joint effort involving Pediatric Cardiac Surgery, General Surgery, and Otolaryngology, division of TEF and repair of esophageal atresia, along with CA repair, was carried out. Via a right thoracotomy, the TEF, located medial to the azygous vein, was skeletonized and ligated. The proximal esophagus was then mobilized up to the thoracic inlet as it coursed through the vascular ring. This enabled esophageal anastomosis with preservation of both aortas. Esophagography on DOL 7 confirmed an intact repair (Figure 2).

The patient remained intubated till DOL 11, at which time she underwent TA repair. Following a standard midline sternotomy and cooling to moderate hypothermia, the LAA was divided and oversewn. The aorta was then transected anteriorly, and the main pulmonary artery (MPA) exiting the posterior aorta was harvested as a single button. The aortic defect from the PA button was repaired with autologous pericardium. Next, through a right ventriculotomy, the previously seen conoventricular septal defect (VSD) was identified and closed. Finally, a 10 -mm pulmonary homograft was anastomosed to the PA bifurcation to complete the repair.

Postoperative echocardiography revealed a satisfactory repair with mild MPA stenosis (Figure 3). Her postoperative course was complicated by failed extubation, requiring tracheostomy, and frequent, persistent atrial ectopy, necessitating prolonged anti-arrhythmic medication. She was discharged on DOL 78 and was noted to be doing well at 1-year follow-up. All procedures performed in studies involving human participants were in accordance with the ethical standards of the institutional and/or national research committee(s) and with the Helsinki Declaration (as revised in 2013). Written informed consent was obtained from the patient's parents.

\section{Discussion}

To our knowledge, this is the first reported case of TA associated with DAA, TEF, and CA. This challenging constellation of pathologies required an interdisciplinary approach involving various specialties, with non- 


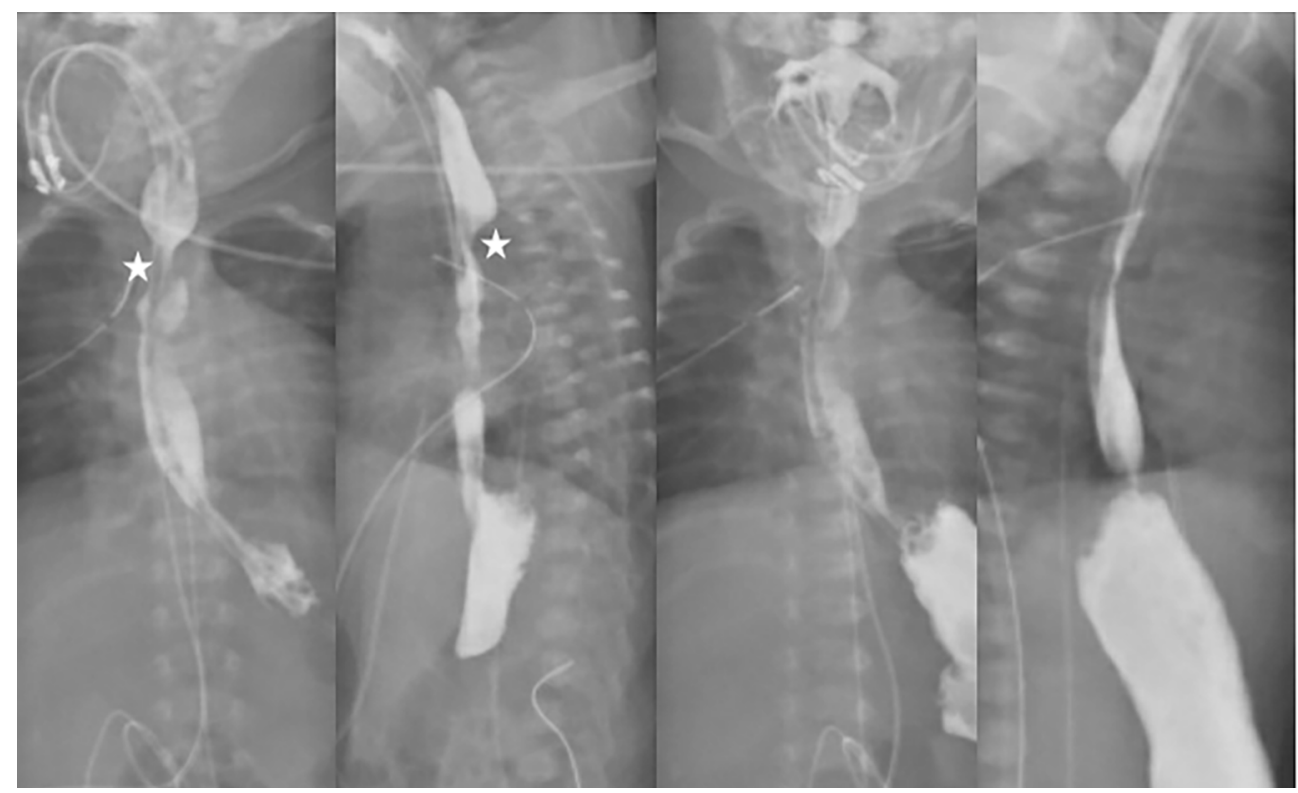

Figure 2 Contrast esophagography following esophageal atresia repair. Single-contrast esophagography under fluoroscopic guidance after tracheoesophageal fistula repair showed no anastomotic leak, as well as marked narrowing of the upper esophagus secondary to the vascular ring (asterisk).
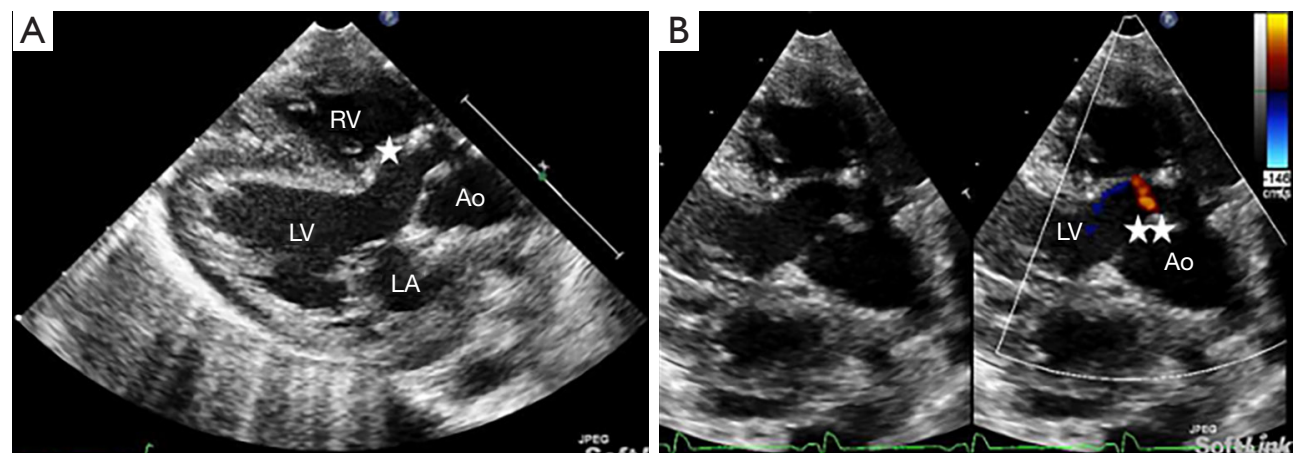

Figure 3 Postoperative Echocardiography. (A) Post-repair echocardiography at one month shows normal bi-ventricular function with an appropriate patch repair of the conoventricular VSD (single asterisk). A small $(\sim 2.0 \mathrm{~mm})$ residual posteroinferior patch margin VSD adjacent to the tricuspid valve was also noted (not visualized here). (B) Mild regurgitation is seen across the truncal valve (double asterisk). Ao, aorta/ common arterial trunk; LA, left atrium; LV, left ventricle; PA, pulmonary artery; RV, right ventricle; VSD, ventricular septal defect.

cardiovascular comorbidities having been shown to increase hospital length-of-stay (LOS), costs, and post-operative morbidity following pediatric cardiac surgery. Numerous studies have documented an exponential, rather than additive, effect of multi-organ disease on post-surgical outcomes, and a comprehensive, team-based strategy that optimizes timing of all repairs is essential for complex cases such as these (3).

Fistula repair in patients with TEF and DAA is usually performed via a right thoracotomy because most of the cardiac mass is located in the left thorax. However, the optimal surgical approach is under continued debate. For one, primary esophageal anastomosis through a right thoracotomy is technically challenging and may be associated with increased postoperative morbidity in patients with a dominant RAA. Although not required in our case, resection of the non-dominant arch is often imperative for adequate esophageal exposure, a task aided 
by the presence of both pediatric cardiac and general surgical teams. Thus, although a left thoracotomy allows excellent exposure of the proximal esophageal pouch, our case validates the feasibility of TEF-complicated-by-DAA repair through a right thoracotomy (4). Furthermore, while the presence of thoracic gastrointestinal malformations in patients with congenital heart disease has been associated with increased post-operative morbidities, including greater LOS and ventilator days, long-term survival seems to be unaffected. In general, such malformations should be corrected prior to repairing the heart defects (5).

Though we successfully repaired the common arterial trunk and DAA during the same procedure, a sequential approach may be appropriate in select cases. The dominance of the RAA requires its preservation during TEF repair. Division of the LAA and vascular ring is better performed via sternotomy at the time of TA repair.

\section{Conclusions}

Our case highlights several key findings. First, when presented with cases of complex anatomy involving numerous organ systems, such as that seen with TA with DAA, tracheoesophageal fistula, and choanal atresia, a multidisciplinary approach to treatment can often be helpful. With input from numerous specialists, such a holistic strategy ensures that the surgical correction of all defects is carried out in the safest manner. In our case, we opted to repair the tracheoesophageal fistula prior to TA repair given the known adverse effects of multi-organ disease on post-neonatal cardiac surgery outcomes. Second, although we chose a right thoracotomy for exposure during fistula repair, the optimal approach (right versus left thoracotomy) in cases such as these remains an enigma, and further analyses are warranted to explore this dichotomy. Finally, the long-term functional and survival outcomes of children with TA and DAA are unknown, and longer-term prospective studies are required to assess the clinical impact of corrective repair in the neonatal period as these patients reach adulthood.

\section{Acknowledgments}

Funding: None.

\section{Footnote}

Reporting Checklist: The authors have completed the CARE reporting checklist. Available at http://dx. doi. org/10. 21037/tp-20-174

Conflicts of Interest: All authors have completed the ICMJE uniform disclosure form (available at http://dx. doi. org/10.21037/tp-20-174). The authors have no conflicts of interest to declare.

Ethical Statement: The authors are accountable for all aspects of the work in ensuring that questions related to the accuracy or integrity of any part of the work are appropriately investigated and resolved. All procedures performed in studies involving human participants were in accordance with the ethical standards of the institutional and/or national research committee(s) and with the Helsinki Declaration (as revised in 2013). Written informed consent was obtained from the patient's parents.

Open Access Statement: This is an Open Access article distributed in accordance with the Creative Commons Attribution-NonCommercial-NoDerivs 4.0 International License (CC BY-NC-ND 4.0), which permits the noncommercial replication and distribution of the article with the strict proviso that no changes or edits are made and the original work is properly cited (including links to both the formal publication through the relevant DOI and the license). See: https://creativecommons.org/licenses/by-nc-nd/4.0/.

\section{References}

1. Imai K, Tsukuda K, Sakazaki H, et al. Persistent truncus arteriosus with double aortic arch and mitral stenosis. Pediatr Cardiol 2013;34:2024-6.

2. Paul JF, Serraf A. Images in cardiovascular medicine. Truncus arteriosus and double aortic arch. Circulation 2002;105:e170.

3. Brown KL, Ridout D, Pagel C, et al. Incidence and risk factors for important early morbidities associated with pediatric cardiac surgery in a UK population. J Thorac Cardiovasc Surg 2019;158:1185-96.e7.

4. Laje P, Martinez-Ferro M. Thoracoscopic double aortic 
arch division with Harmonic scalpel: a case report. J

Thorac Cardiovasc Surg 2007;133:819-20.

5. Mery CM, De Leon LE, Rodriguez JR, et al. Effect of
Gastrointestinal Malformations on the Outcomes of Patients With Congenital Heart Disease. Ann Thorac Surg 2017;104:1590-6.

Cite this article as: Sengupta A, Murthy RA, Pastuszko P. Staged repair of truncus arteriosus with double aortic arch, tracheoesophageal fistula, and choanal atresia via a multi-disciplinary approach: a case report. Transl Pediatr 2021;10(2):454-458. doi: 10.21037/tp-20-174 